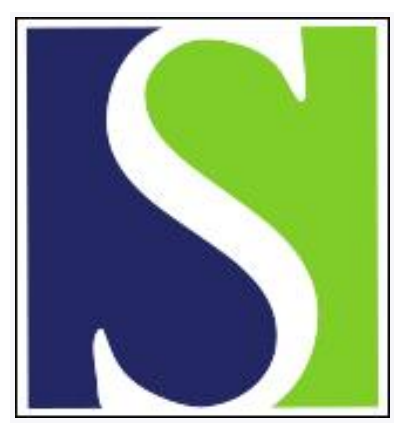

Scand J Work Environ Health 1998;24(6):445-448

https://doi.org/10.5271/sjweh.368

Issue date: Dec 1998

Pesticides and congenital malformations - how many studies will it take to reach a conclusion?

by Kogevinas M, Sala M

This article in PubMed: www.ncbi.nlm.nih.gov/pubmed/9988086

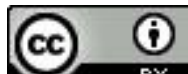




\section{Pesticides and congenital malformations - how many studies will it take to reach a conclusion?}

Pesticides and congenital malformations is a long-standing story. A recent review identified 34 epidemiologic studies on occupational exposure to pesticides in relation to congenital malformations published after 1980 (1). There are no conclusions, however, to be drawn from the conjunct of the data even though some of the studies were not small in size and a few used fairly detailed methods for exposure assessment. One study on paternal exposure to pesticides and congenital malformations is even published in this issue (2). The evaluation of exposure to specific pesticides was carefully validated and is more detailed than in most studies in this field. Some positive associations are reported, but, still, there are no firm conclusions to be drawn from this study. This feature does not, in any way, disqualify the results of this well conducted study. Firm conclusions would rarely be drawn from a single study, and they usually require a complete set of convincing evidence from epidemiologic research, chronic bioassays, and toxicologic and other laboratory studies. One question which remains, however, after the publication of this study is "How much more do we know?" If 10 or 20 more studies similar to this one were available, would we be able to draw firm conclusions? It is difficult to say, but probably we would not. There is even little hope that a meta-analysis could provide systematic answers given the wide heterogeneity in design and exposure between studies.

Part of the problem lies with the disease(s). The category congenital malformations includes a wide variety of different pathologies. Many, but not all, are rare and some may be difficult to diagnose and register. Some agents may also produce spontaneous abortions that consequently are never diagnosed or registered as malformations, and some malformations may be detected through prenatal screening that leads to induced abortions. Furthermore, little is known on the environmental (nongenetic) causes of most malformations. A common problem in studies evaluating environmental exposures is that, because of small numbers, they tend to examine the malformations jointly $(1,2)$. It is, however, largely unknown whether malformations such as hypospadias, oral clefts, hydrocephaly, Down syndrome, limb reduction defects, septal heart defects, or others share many causal agents. The net effect of evaluating all congenital malformations together or using large heterogeneous groups (eg, heart defects) is that of gross misclassification and consequent biased estimates.

Misclassification in the assessment of exposure to pesticides constitutes another important part of the problem, one not specific to the investigation of congenital malformations. Studies on congenital malformations involve the following difficulties: (i) exposure information has to be collected for two persons (mother and father) rather than one, (ii) specific agents may be acting in different time windows of exposure, (iii) the time windows may be narrow and not lasting more than a few weeks, (iv) users of pesticides tend to be exposed to mixtures rather than to a single pesticide, and (v) in general population studies it is difficult to identify wide exposure contrasts to pesticides. These problems are accentuated when exposure information is collected retrospectively.

It usually takes years to identify and provide convincing evidence for causal relationships. The simple accumulation of studies using valid research methodologies frequently suffices for conclusions to be drawn, as seems to be increasingly the case, for example, with chlorination by-products in drinking water and cancer (3). This process, however, does not seem to be happening with research on pesticides and malformations. The case of the carcinogenicity of dioxins serves as a different example. It took about 20 years of research and tens of epidemiologic studies to establish that dioxins are 
carcinogenic to humans (4). The turning point was the acceptance that different types of studies than those available at the time were necessary. Large cohort studies of heavily exposed subjects were initiated and new analytical techniques allowing the detection of individual dioxin blood levels were used to develop convincing exposure models. The impasse in research in various fields led epidemiologists to pursue different, frequently complex and expensive approaches which would have been considered unrealistic in the past $(5,6)$. These efforts frequently coincided with the availability of new epidemiologic and, mostly, new laboratory techniques. Whether such efforts will offer conclusive answers remains to be seen. What makes these approaches attractive, however, is that they attempt to overcome the major deficiencies of past epidemiologic research, rather than replicating it. It may be time to consider a similar type of action to try and elucidate the relationship between specific environmental exposures and specific reproductive outcomes.

The evaluation of whether occupational exposure to pesticides is associated with risk for congenital malformations implicitly poses the issue of whether the widespread low-level exposure to these compounds in the general population is also associated with an excess risk. In tackling this question, a key issue is why have we been so ineffectual in identifying environmental causes of congenital malformations and in identifying the reasons for the apparent increasing incidence of malformations such as hypospadias. While continuing (frequently inefficiently) to pursue very specific environmental agents associated with congenital malformations, we may not have been perceptive of the more generic insults to reproductive biology posed by the chemicalization of our environment. Although international studies are being conducted (7), much of our effort has gone into within-population studies of individual-level risk factors. Yet much of the explanation may have to do with shifts in background conditions, as would have been a gradual generalized increasing exposure to chemicals - including pesticides - with hormonal action. Such changes may not even be "visible" to classical epidemiologic designs and may require a more "ecological" approach that is somewhat outside the field-of-vision of mainstream epidemiology (8).

A final option that epidemiologists should consider is to wait — wait until other areas of biomedical research allow significant improvements in the assessment of exposure to pesticides, the diagnosis and classification of congenital malformations, or a better understanding of the disease mechanisms. Waiting should not be perceived as a call for passive attitudes or as a call for an abandonment of difficult areas of research, such as that examining pesticides (and other environmental agents) and congenital malformations. It is merely a recognition that epidemiologic research is not always the most appropriate, innovative, or efficient means for evaluating human risk. Neither does waiting mean that precautionary action should not be taken to control human exposures, in view of the potential, but still unproved adverse effects.

A few months earlier the Scandinavian Journal of Work, Environment \& Health published an editorial on another area of research (9), and this editorial carried essentially the same message. Epidemiologists cannot guarantee clear positive or negative results before doing a study. When, however, they embark upon a research project, they should try hard to develop testable hypotheses and evaluate them adequately so that conclusive answers can be drawn at some reasonable point in time. To answer the title question, it is not a matter of how many but, rather, of what type of studies we need.

\section{References}

1. Garcia AM. Occupational exposure to pesticides and congenital malformations: a review of mechanisms, methods and results. Am J Ind Med 1998;33:232-40.

2. Garcia AM, Benavides FG, Fletcher T, Orts E. Paternal exposure to pesticides and congenital malformations. Scand J Work Environ Health 1998;24(6):473-80.

3. Cantor KP. Drinking water and cancer. Cancer Causes Control 1997;8:292 - 308.

4. International Agency for Research on Cancer (IARC). Polychlorinated dibenzo-para-dioxins and polychlorinated diben- 
zofurans. Lyon: IARC, 1997. IARC monographs on the evaluation of carcinogenic risks to humans, vol 69.

5. Alavanja MCR, Sandler DP, McMaster SB, Zahm SH, McDonnell CJ, Lynch CF, et al. The agricultural health study. Environ Health Perspect 1996;104:362 -9.

6. Riboli E. Nutrition and cancer: background and rationale of the European Prospective Investigation into Cancer and Nutrition (EPIC). Ann Oncol 1992;3:783--91.

7. Stoll C, Ayme S, Beckers R, Calzolari E, Cornel M, Cushieri A, et al. Distribution of single organ malformations in European populations: EUROCAT Working Group. Ann Genet 1995;38:32—43.

8. Susser M. The logic in ecological, II: the logic of design. Am J Public Health 1994;84:830 - 5

9. Hernberg S. Inconclusive cancer epidemiology [editorial]. Scand J Work Environ Health 1998;24:161 _- 4.

Manolis Kogevinas, MD, \& Maria Sala, MD

Respiratory and Environmental Health Research Unit

Institut Municipal d'Investigació Mèdica (IMIM)

80 Doctor Aiguader Rd

Barcelona 08003, Spain

[E-mail:Kogevinas@imim.es] 